\title{
Towards an Error Model for OpenMP
}

M. Wong, M. Klemm, A. Duran,

T. Mattson, G. Haab, B. R. de Supinski,

A. Churbanov

March 24, 2010

6th International Workshop on OpenMP

Tsukuba, Japan

June 14, 2010 through June 16, 2010 
This document was prepared as an account of work sponsored by an agency of the United States government. Neither the United States government nor Lawrence Livermore National Security, LLC, nor any of their employees makes any warranty, expressed or implied, or assumes any legal liability or responsibility for the accuracy, completeness, or usefulness of any information, apparatus, product, or process disclosed, or represents that its use would not infringe privately owned rights. Reference herein to any specific commercial product, process, or service by trade name, trademark, manufacturer, or otherwise does not necessarily constitute or imply its endorsement, recommendation, or favoring by the United States government or Lawrence Livermore National Security, LLC. The views and opinions of authors expressed herein do not necessarily state or reflect those of the United States government or Lawrence Livermore National Security, LLC, and shall not be used for advertising or product endorsement purposes. 


\title{
Towards an Error Model for OpenMP
}

\author{
Michael Wong, ${ }^{1}$ Michael Klemm, ${ }^{2}$ Alejandro Duran, ${ }^{3}$ Tim Mattson, ${ }^{2}$ Grant Haab, ${ }^{2}$ \\ Bronis R. de Supinski, ${ }^{4}$ and Andrey Churbanov ${ }^{2}$ \\ ${ }^{1}$ IBM Corporation $\quad{ }^{2}$ Intel Corporation \\ ${ }^{3}$ Barcelona Supercomputing Center $\quad{ }^{4}$ Lawrence Livermore National Laboratory \\ michaelw@ca.ibm.com, alex.duran@bsc.es,bronis@llnl.gov \\ \{michael.klemm,timothy.g.mattson\}@intel.com \\ \{grant.haab, andrey.churbanov\}@intel.com
}

\begin{abstract}
OpenMP lacks essential features for developing mission-critical software. In particular, it has no support for detecting and handling errors or even a concept of them. In this paper, the OpenMP Error Model Subcommittee reports on solutions under consideration for this major omission. We identify issues with the current OpenMP specification and propose a path to extend OpenMP with error-handling capabilities. We add a construct that cleanly shuts down parallel regions as a first step. We then discuss two orthogonal proposals that extend OpenMP with features to handle system-level and user-defined errors.
\end{abstract}

\section{Introduction}

OpenMP [13] is a wide-spread and well-known programming model for parallel programming on shared memory platforms. OpenMP's initial focus was to provide portable parallel programming for High Performance Computing (HPC) platforms. OpenMP's expressive programming model, including support for incremental parallelization, has led application programmers in other areas (e.g., enterprise software) to consider it for their applications. However, OpenMP's lack of any concept of errors or support to handle them has prevented wide-spread adoption of OpenMP by industries.

OpenMP 3.0 only requires an implement to provide best effort execution for runtime errors. Application are often terminated and users must restart. While perhaps tolerable even if undesirable for HPC users, it is clearly unacceptable to terminate enterprise applications. Thus, programmers must implement workarounds (such as those in Section 2) that make development and maintenance more difficult and often prevent key compiler optimizations.

In this paper, we present the current plans of the OpenMP Error Model Subcommittee to provide error handling extensions. Clean semantics for errors raised in concurrent code paths are non-trivial $[7,17,19]$ so we do not focus on solutions to concurrent errors. We instead consider mechanisms to detect and to respond to errors (both OpenMP runtime and user code errors). We provide our criteria and limitations for OpenMP error proposals in Section 3. We then propose a two-phase process to add error support in Section 4. We plan to add a done construct that cleanly terminates an OpenMP region to the next OpenMP version while our longer term strategy is considering two proposals of catching and handling OpenMP runtime and user-defined errors. 


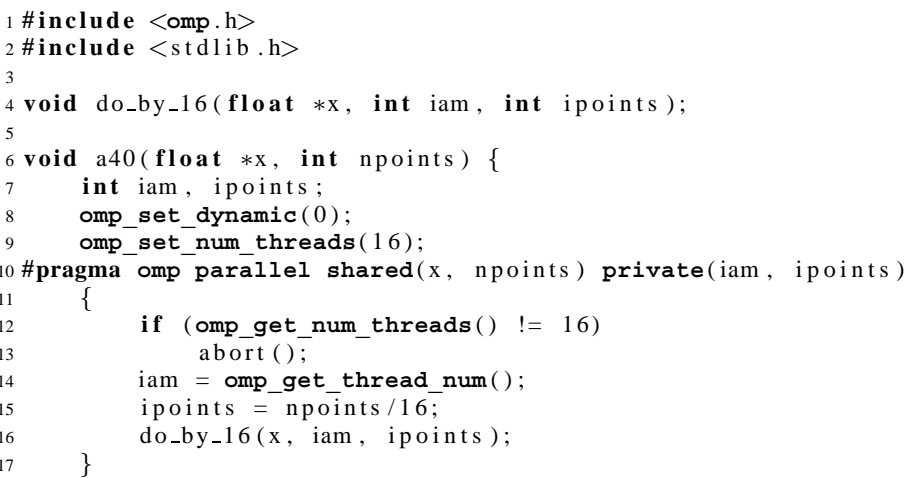

Fig. 1. Setting and checking the threads count for parallel regions

\section{Current State in OpenMP Error Handling}

This section motivates the need for clean error handling semantics in OpenMP. We first investigate the current OpenMP specification's error handling requirements for errors that arise within OpenMP implementations. We then turn to OpenMP's features for handling user-defined errors, i. e., C++ exceptions and return codes in $\mathrm{C}$ and Fortran.

\subsection{OpenMP runtime errors}

OpenMP has never offered clean semantics to handle errors that arise within OpenMP runtime implementations. Fig. 1 shows Example 40.1.c, which we chose arbitrarily from the OpenMP 3.0 specification [13]. We use this example to investigate potential errors that arise and how OpenMP deals with them.

The code in Fig. 1 calls omp_set_dynamic (line 8) and omp_set_num_threads (line 9) to ensure that exactly 16 threads execute the following parallel region. OpenMP does not prescribe how an OpenMP implementation should react if a programmer passes inconsistent values (e. g., a negative thread count) to these functions. Any behavior, from terminating the application to using any (valid) value is compliant.

If the OpenMP runtime cannot supply the requested number of threads (e. g., due to resource constraints) for the parallel region, OpenMP does not prescribe how the implementation must react. For instance, it can terminate the program with (or without) an error message or continue with an arbitrary thread count. Thus, programmers must explicitly check the thread count by calling omp_get num_threads and take appropriate actions; in this example, the program explicitly aborts (line 13) if it does not get exactly 16 threads.

Other errors can occur when the OpenMP runtime creates the threading environment and allocates resources for the parallel region. The OpenMP specification allows the OpenMP implementation to define how it responds to these errors. Hence, programmers cannot portably intercept potential errors in order to take more appropriate actions. 


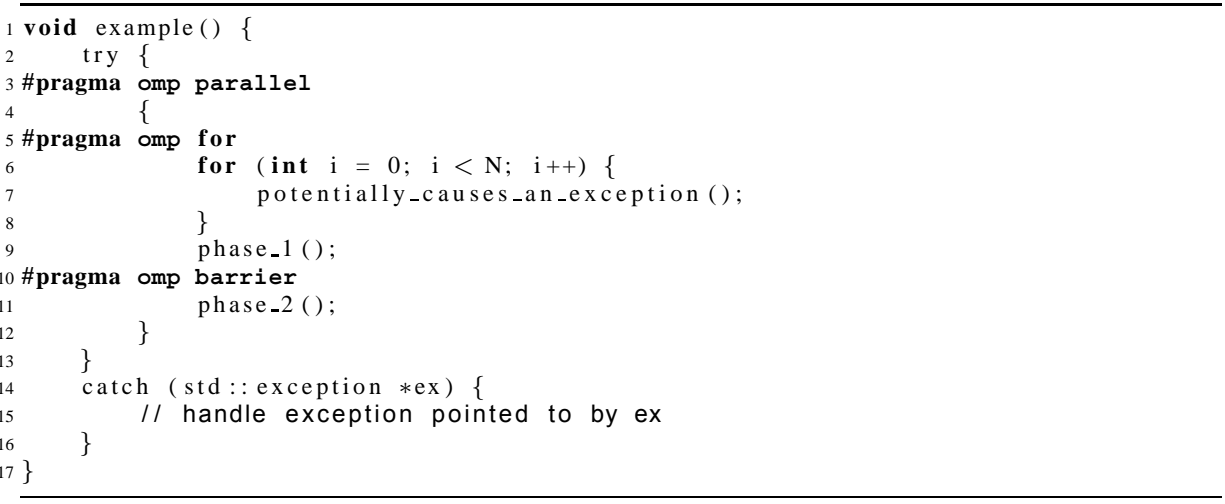

Fig. 2. Non-conforming OpenMP code

OpenMP runtime support routines also have no defined error semantics. The specification does not prescribe actions for an OpenMP implementation if routines fail, which is also true if users supply incorrect values for OpenMP environment variables. These unspecified behaviors complicate the implementation of resilient applications, which must continue functioning in the presence of errors or unexpected conditions.

\subsection{User-defined errors}

Most mission-critical applications cannot silently ignore errors and continue execution. Thus, error handling consumes significant application development time [15]. We now discuss how OpenMP applications must handle $\mathrm{C}++$ exceptions; we cover $\mathrm{C}$ and Fortran error handling patterns at the end of this sub-section.

Sequential C++ codes usually map errors to exceptions that are thrown where the error arises and caught by error handling code. Fig. 2 shows a simple program skeleton that does not conform to the OpenMP specification if an exception arises in the for construct. Several threads that execute could raise an exception and concurrently multiple might occur. Any exception would cause a premature termination of the parallel region, which violates the Single-Entry Single-Exit (SESE) principle that is required to all OpenMP parallel regions including those that use longj mp ( ) or throw [13]. Exceptions also must not escape any worksharing region or critical or master section. Thus, applications must catch all exceptions thrown within any structured block that is associated with an OpenMP construct before the block is exited. A throw that is executed inside an OpenMP region must cause execution to resume within the same region and the same thread that threw the exception must catch it.

Fig. 3 shows how to handle exceptions in a parallel region correctly by catching exceptions (line 17) so that they do not escape an OpenMP construct. For simplicity, we assume that all potential exceptions inherit from $\mathrm{C}++$ standard exception class std: : exception. We use a shared variable to notify other threads that the exception occurred. As we cannot prematurely terminate the for construct (the OpenMP specification prohibits changing the loop control variable or using a break statement), we 


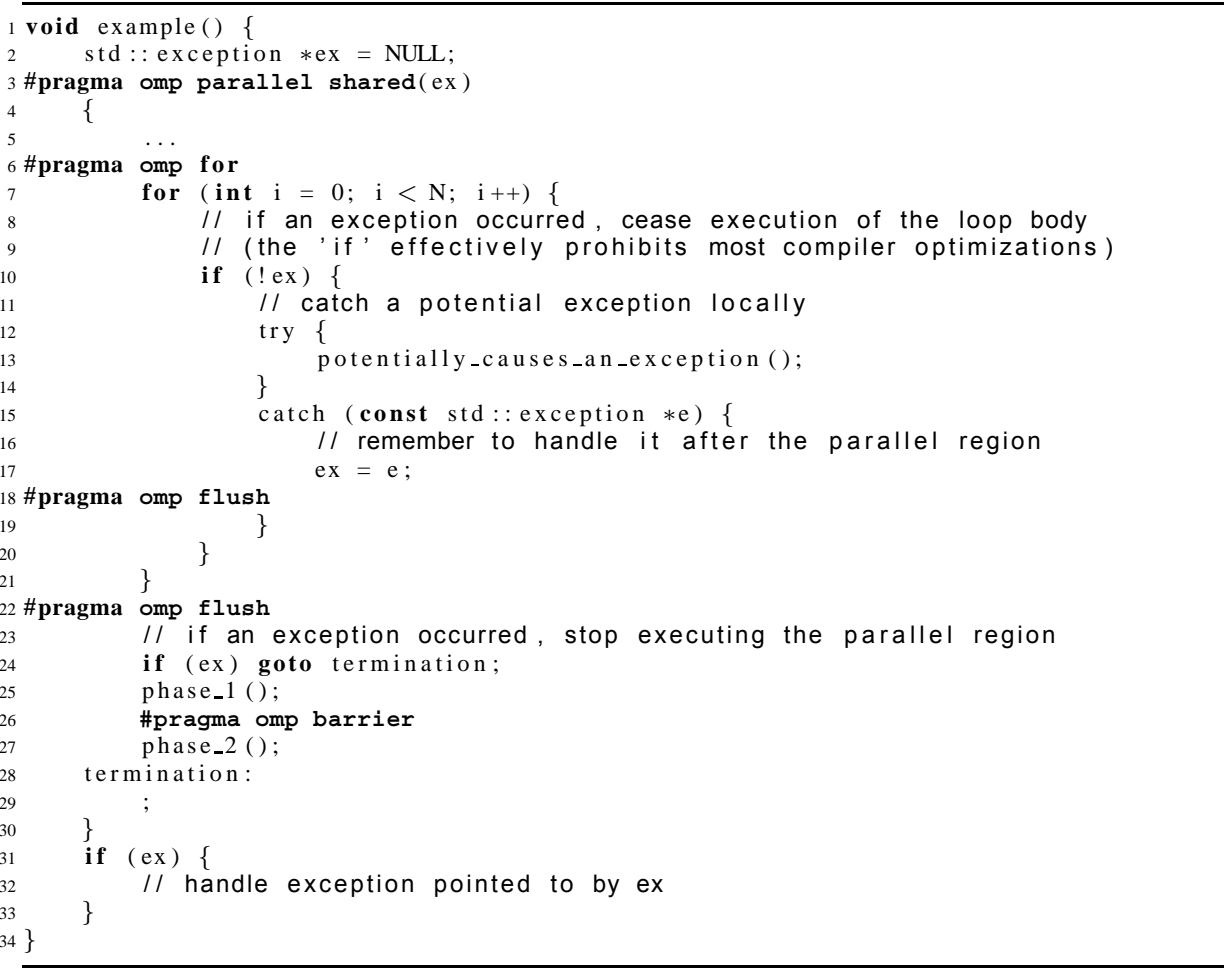

Fig. 3. Shutting down an OpenMP-parallel region in presence of an exception

use the exception flag to skip the remaining loop body when an exception occurs; this conditional if disables many standard compiler optimizations such as vectorization. Thus, the compliant code will run slowly even if no exceptions arise. We check the flag again before phase_1 () is executed to ensure that we skip line 25 branching to the termination label (line 28). We again check the flag (line 31) following the parallel region to handle the exception consistently with the sequential semantics.

While Fig. 3 provides a method to terminate a parallel region, worksharing constructs and tasks require conditional tests to skip the remainder of the structured block. Synchronization constructs such as critical can use the C++ RAII (Resource Acquisition is Initialization) idiom with scopes locks to ensure they are properly released. However, we know of no suitable workarounds for the sections and ordered constructs.

Most applications in $\mathrm{C}$ and Fortran indicate errors with special return values or an errno variable (e. g., POSIX return codes [18]) or through an additional function argument (e.g., MPI error codes [11]). Programmers must check these manually to determine if an error has arisen and handle it similar to the coding pattern shown in Fig. 3. Nonetheless, OpenMP SESE requirements often cause substantial changes to the sequential coding pattern in these languages. 


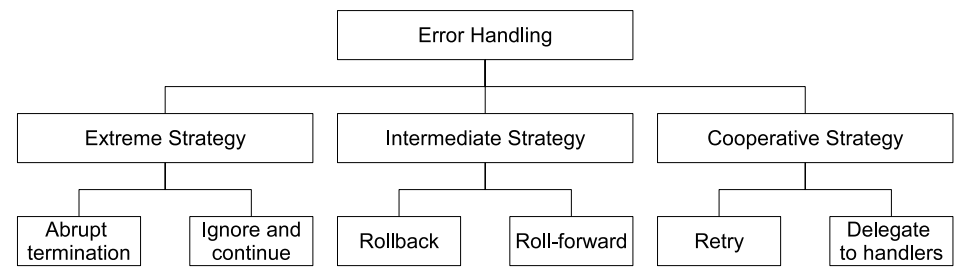

Fig. 4. Classification of error handling strategies

\section{Design Objectives}

We now discuss the major requirements for the cleaner error handling semantics that OpenMP clearly needs. First, the new error model must support all OpenMP base languages and allow for additional ones consistently with the OpenMP philosophy of incremental parallelization. Thus, it must support the methods of exception-aware (e.g., $\mathrm{C}++$ ) and exception-unaware (e. g., $\mathrm{C}$ and Fortran) languages. The error handling facilities must tightly integrate modern exception handling while supporting traditional techniques based on status flags and return values in order to avoid significant changes to the sequential code.

Second, the new error model must provide exception-aware support even for exception-unaware languages since they have cleaner, stronger semantics than classic mechanisms (e.g., error codes). Programs cannot ignore (or forget to detect) exceptions, which always force the programmer to respond in some way. Return codes intermix data and errors, while exceptions decouple error handling from the primary application functionality. Finally, exceptions simplify the reuse of error handling code and eliminate repetitive, error-prone code since programmers do not have to locate error handlers where errors arise.

The new model must support system-level errors a well as user-defined ones. Ideally, an OpenMP implementation must provide notification of errors that arise within the runtime system without requiring special checks. Further, the model must not require the programmer to distinguish between system-level and user-defined errors although it should allow it. Instead, programmers should rely on a single, common framework that can handle errors from either source.

The model must be flexible and provide tools to implement different error handling strategies. Fig. 4 classifies error-handling mechanisms into different categories. Our goal is to support the extreme strategy and cooperative strategy. Intermediate strategies are beyond the scope of our working group. However, they could be implemented through transactional memory [9], which is a possible OpenMP extension [10].

Most importantly, the error handling features must provide backward compatibility. It cannot break existing codes that have adapted to the current "best effort" errorhandling requirements. It also must not require new applications to handle errors if they do not involve mission-critical computations. 


\section{OpenMP Error Handling Proposals}

We propose a two-phase plan to satisfy the error-model requirements. Our first step will provide a construct to terminate OpenMP regions, which directly supports the abrupt termination pattern for user-defined errors of Fig. 4. Section 4.1 describes the done construct that the OpenMP Error Model Subcommittee plans to include in the OpenMP 3.1 specification.

Our second step will support the ignore and continue, retry and delegate to handlers strategies. We describe the two orthogonal proposals that the OpenMP Error Model Subcommittee is considering in Section 4.2 and Section 4.3. We discuss the implications of these mechanisms, for which we target OpenMP 4.0, with a specific focus on backwards compatibility.

\subsection{The done construct}

Our proposed done construct terminates innermost OpenMP regions, which provides initial support for user-defined errors (particularly for $\mathrm{C}$ and Fortran). We build on prior proposals to terminate parallel regions that were considered for the OpenMP 1.0 specification. HP's compiler still supports a limited pdone construct [1]. The done construct reflects the OpenMP philosophy of incremental parallelization through compiler directives, unlike the alternative of a new runtime function, which would alter the underlying sequential code or require conditional compilation guards.

The done construct has the $\operatorname{syntax}^{1}$

1 \#pragma omp done [clause-1ist ]

with clause-list being one or more of if (expr), ${ }^{2}$ parallel, for $(\mathrm{C} / \mathrm{C}++)$, do (Fortran), sections, single, or task. The binding set of the done construct is the current thread team. It applies to the innermost enclosing OpenMP construct(s) of the types specified in the clause (i. e., parallel or task).

The done construct causes the encountering thread to terminate the subset of the innermost parallel, worksharing and task regions that correspond to the specified clause. It conceptually sets a cancellation flag that the other team members must evaluate at no later than the next cancellation point that they execute. Hence, other threads may not immediately terminate execution when a thread encounters the done construct. This delayed termination allows more efficient execution, as the mechanism does not require interrupts or frequent polling of the cancellation flag.

We make the set of cancellation points implementation defined in order to avoid restricting implementation choices although we are exploring a minimal set. Efficient implementation of the done construct will likely require different cancellation points under different OpenMP implementations. A minimal set of cancellation points could be: entry and exit of regions, barriers, critical sections, completion of a loop chunk and calls to runtime support routines.

\footnotetext{
${ }^{1}$ We present the $\mathrm{C} / \mathrm{C}++$ syntax only; the Fortran syntax is similar and can easily be derived.

${ }^{2}$ The if clause has the same semantics as the if clauses of other constructs.
} 


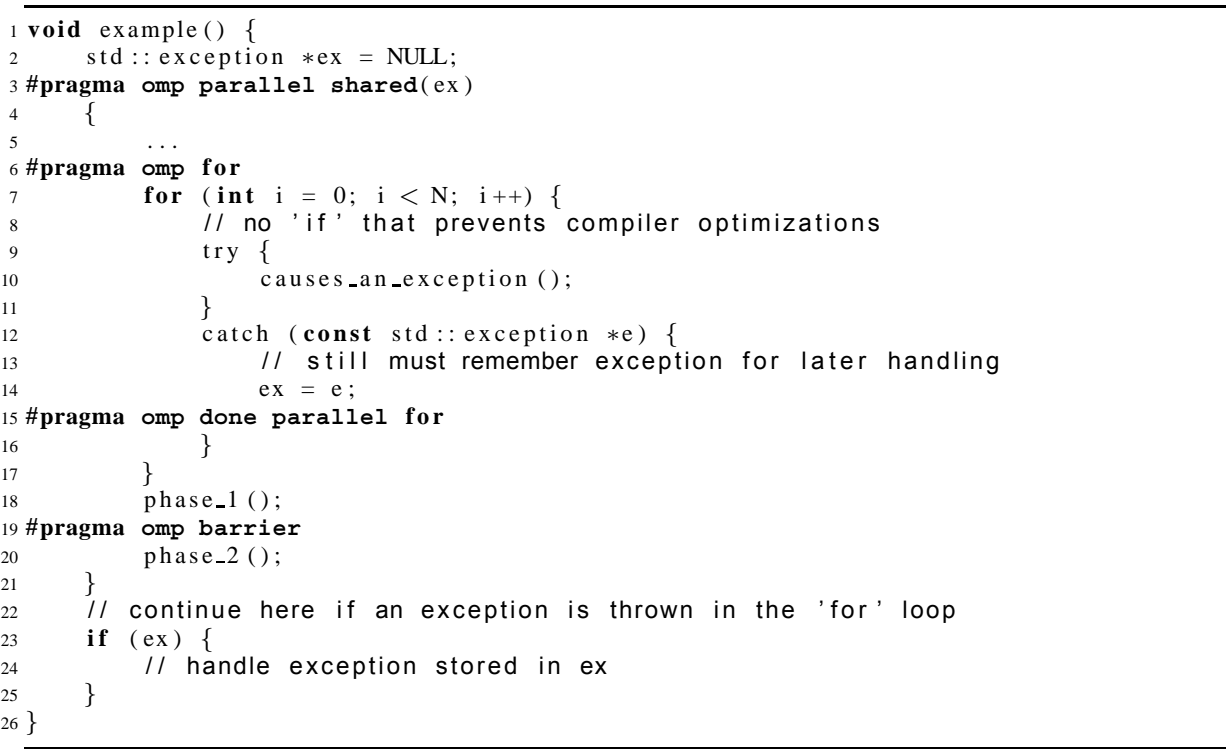

Fig. 5. Shutting down an OpenMP parallel region with the done construct

The done construct supports elegant and robust termination of OpenMP parallel execution, as Fig. 5 shows. If an exception is raised during execution of the worksharing construct spanning lines $6-17$, the catch handler can trigger a done parallel for to shut down the worksharing construct safely. The for clause terminates the worksharing construct while the parallel clause terminates the parallel region. Execution continues at the if in line 23 after termination of the regions. While the done construct cleanly terminates OpenMP regions, the programmers must still track the exceptions through pointers and apply the sequential handler. However, there is no need for the tricky flush as the exception pointer is not accessed within the parallel region.

\subsection{Modest proposal based on return codes}

In the long term, we must provide features that support the existing error-handling code in exception-unaware languages. We must also ensure backwards compatibility for existing OpenMP programs, which limits the mechanisms and API choices for our errorhandling model.

Thus, we consider the minimal functionality that an error-handling system requires. An OpenMP error-handling mechanism must:

- Communicate to the user program that an error has occurred;

- Provide sufficient information to identify the type and source of the error.

- Support execution after the error arises with well-defined program state so that the program can respond and continue. 
We now discuss a modest error-handling proposal based on return codes that meets these requirements.

In order to support continued execution, we require that the program continues at the first statement following the end of the innermost construct when an error occurs inside any OpenMP construct. Any variables that are created or modified inside the construct have an undefined value.

We communicate the error condition to the program through a variable that is shared between the members of the thread team. The omp-error-var variable is of type omp_error_t and stores an error code that identifies whether any thread that executed the preceding OpenMP construct or runtime library routine encountered an error and, if so, the error's type. If concurrent errors occur, the runtime system may arbitrarily select one error code and store it in the shared variable.

Programs can query the value of this variable by calling a new OpenMP runtime support routine:

1 omp_error_t omp_get_error(char $*$ omp_err_string, int bufsize)

This function can return any value of a set of constants that are defined in the standard OpenMP include file. While implementations can support additional error codes, we anticipate a standard set that may include values such as:

- OMP_ERR_NONE

- OMP_ERR_THREAD_CREATION

- OMP_ERR_THREAD_FAILURE

- OMP_ERR_STACK_OVERFLOW

- OMP_ERR_RUNTIME_LIB

The omp_get_error function in addition returns an implementation-defined, zeroterminated string in the memory area pointed to by omp_err_string. In the string, an implementation may provide more information about the type and source of the error.

Fig. 6 shows an example that uses our error code handling proposal. While the code is less elegant than approaches based on exceptions and callbacks, we can add this solution to any OpenMP base language and satisfy the backward compatibility requirement. This error code approach is more straightforward in that it does not introduce complex execution flows through error handlers. This directly resembles the characteristics of error handling in $\mathrm{C}$ and Fortran.

\subsection{The callback error handling mechanism}

The next longer term proposal is our callback error handling proposal. It provides many of the benefits of exception-aware languages to $\mathrm{C}$ and Fortran. This proposal, which slightly extends a previous callback-based proposal by Duran et al. [3], achieves its functionality through callback notifications and supports both exception-aware and exception-unaware languages. Hence, it supports all OpenMP base languages.

The prior proposal extends OpenMP constructs with an onerror clause that overrides OpenMP's default error-handling behavior, as line 8 of Fig. 7 shows. Programmers specify a function that is invoked if any errors arise (lines 1-4) within the OpenMP implementation (e. g., directives and API calls). The handler can take any necessary actions and notify the OpenMP runtime about how to proceed with execution (e. g., retry, 


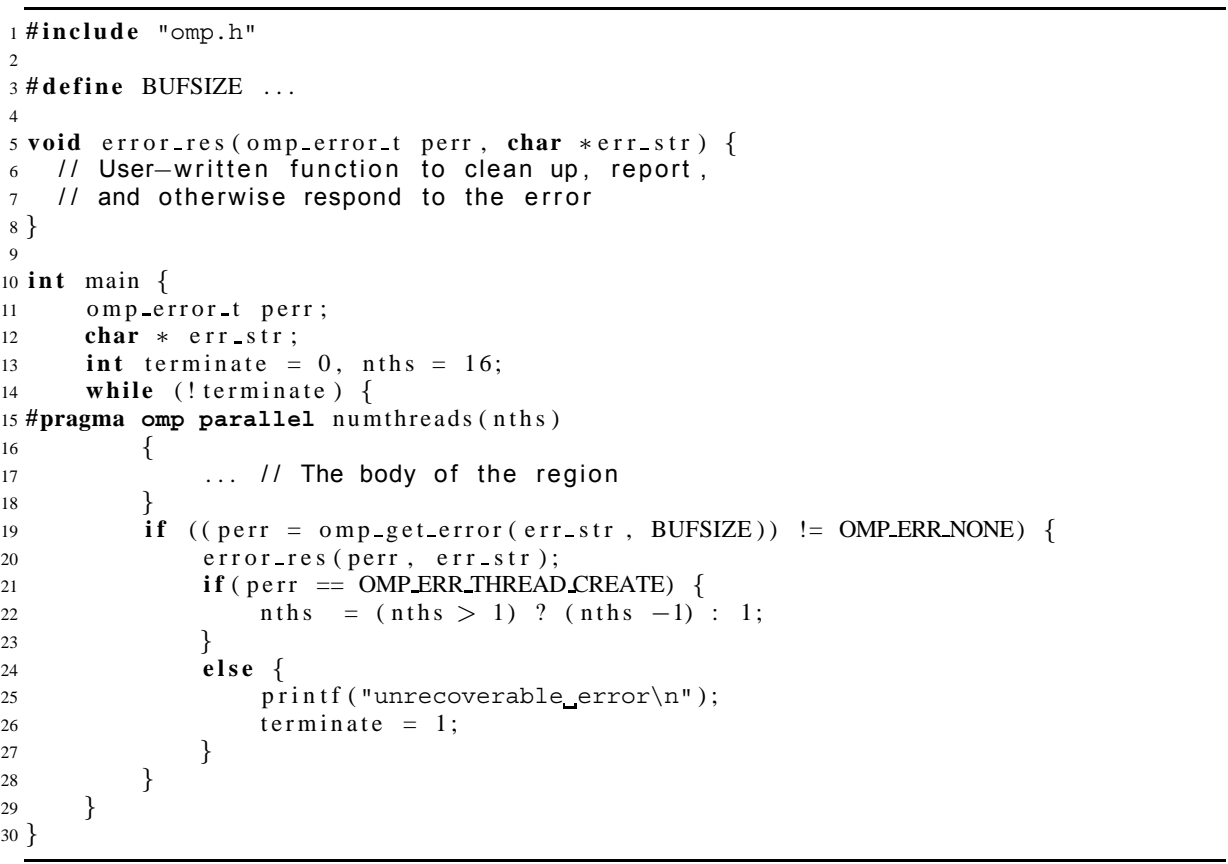

Fig. 6. Using the proposal based on error codes

abort, or continue). The prior proposal also provided a set of default handlers that the program can specify with the onerror clause to implement common error responses. Also, the context directive associates error classes and error handlers with sequential code regions to support errors that arise in OpenMP runtime routines. Users are not required to define any callbacks in which case the implementation will provide backward compatibility with the current best effort approach.

Our callback proposal extends the onerror proposal to meet our OpenMP errorhandling model requirements. We add the error class OMP _USER_CANCEL to associate error handlers with termination requests of done constructs, which supports voluntary region termination and, thus, user-defined error handling. We provide the error class OMP_EXCEPTION_RAISED, so that error handlers can catch and handle C++ exceptions, either locally or globally by re-throwing. Thus, this mechanism supports the exception-aware semantics of $\mathrm{C}++$ that handle and re-throw exceptions. Finally, we are exploring extensions such as specifying a default handler with an environment variable so that applications can take appropriate actions for errors that occur during initialization of the OpenMP runtime or from invalid states of internal control variables.

\section{Other Concurrent Programming Error Handling Models}

POSIX threads (pthreads) [5] is one of the earliest concurrent programming models. It specifies a binding for $\mathrm{C}$, which all vendors have reused for $\mathrm{C}++$ and Fortran. An 


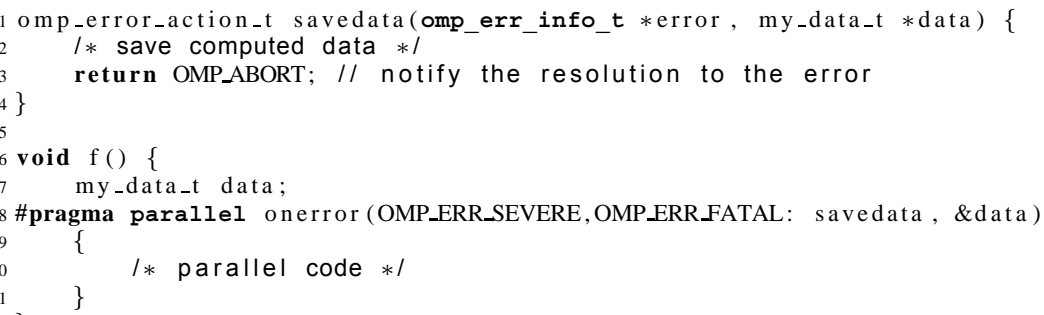

Fig. 7. Example of OpenMP error handling using the callback proposal

effort is currently underway to define a POSIX threads binding for C++ [16]. Four error handling models are commonly used for threads; two apply to pthreads.

The first, and the simplest pthreads model, stops a thread when an error occurs using pthread_kill or pthread_cancel for asynchronous mode. This method unconditionally stops a thread and provides no mechanism to respond by calling cleanup actions. The Java Thread API [12] includes a similar facility, Thread.destroy or Thread. stop. This model was reasonable for exception-unaware languages but does not support exception-aware languages. It could corrupt a running program because a thread may be partially completed, leaving some object or data in an incomplete state. Although incomplete, any programming model should support this simplest termination error handling model, which is why we add the done construct.

The second model allows the target thread to delay its response to a termination request. The many examples of this model all use the idea of an interruption or cancellation point that is a well-defined point at which the target thread must respond. The full list of pthreads interruption points include calls to wait (), sleep (), create () and write (). In addition, cancellation points are usually encountered when a thread is blocked while sleeping, joining another thread or task, or waiting for a mutex, semaphore signal or synchronization. When a thread requests another thread to cancel itself (with pthreads_cancel in pthreads), the request is mapped in the target thread to an exception that is checked at cancellation points and can be rethrown or handled. With pthreads, a program can install a chain of cancellation handlers, which serve a similar purpose as destructors in $\mathrm{C}++$.

With pthreads, cancellation requests cannot be ignored or caught: the target must stop at its next cancellation point and cancellation cannot be stopped once it has begun. While acceptable for $\mathrm{C}$ or Fortran which lack exceptions and object destructors, exception-aware languages like $\mathrm{C}++$ require the ability to catch and recover from errors and to continue correct execution. Since an OpenMP error handling model must support $\mathrm{C}++$, OpenMP requires a richer model than pthreads.

The third model, a (partially) cooperative model, implements error interrupts as exceptions thrown by wait/join/sleep calls. Like pthreads, the target thread can let destructors unwind the stack and exit. Unlike pthreads, the target thread can choose to unwind its stack until it finds a handler that catches and handles the exception, and then resume normal operation. Alternatively, it can catch the exception immediately and 
ignore it. Thread. interrupt in Java and Thread. Interrupt in .NET provide a partial cooperative model, while one is currently being prepared in C++0x [4].

The fourth model, a fully cooperative model, allows the target thread to check whether it is the target of an interrupt anywhere, not just at cancellation points. This cooperative model is planned for $\mathrm{C}++0 \mathrm{x}$.

MPI supports error handling with a callback mechanism [11]. This mechanism provides a default behavior of aborting when errors occur within MPI as well as an additional predefined handler that allows errors to return an error code. MPI error support is similar to our overall proposal although the issues are simpler since MPI defines error handling as a local operation other than with the default abort behavior.

Michael Süß [14] proposes a cancellation proposal with a pragma-based user technique for stopping threads. This proposal does not define any cancellation points, and is generally considered a cooperative exception approach. It is also limited to threads and regions and cannot shutdown other constructs, e. g., tasks or subteams. Our done construct is similar but simpler to use and covers all existing OpenMP constructs.

OpenMP/Java [8], JCilk [2], and TBB [6] support exception handling by setting an internal flag. OpenMP/Java and JCilk check this flag at cancellation points, terminate parallel execution, and rethrow exceptions to the sequential code. Both languages arbitrarily select one exception if multiple arise. TBB registers the first exception and cancels the task group; it ignores other concurrent exceptions. TBB supports exception propagation for $\mathrm{C}++0 \mathrm{x}$ to pass exceptions to other threads. All three models lack a flexible mechanism to react to error situations other than terminating parallel execution. With our proposal we strive to provide a toolbox of error-handling mechanisms that help programmers implement more sophisticated error-handling strategies.

\section{Conclusions and Future work}

We have presented the current directions that the OpenMP Error Model Subcommittee is pursuing. OpenMP currently has no concept of errors. We have identified the requirements of OpenMP error-handling models. The most important requirements are the need to support all OpenMP base languages and to provide backward compatibility for applications that assume no error-handling support is available. The first requirement mean that the mechanism must not require significant changes to sequential code for exception-unaware and exception-aware languages.

We have detailed planned error-handling extensions for future OpenMP specifications. Our plans include the standardization of a done construct that supports termination of OpenMP regions, which not only supports error handling but will also prove useful for some task-based programs. We anticipate that OpenMP 3.1 will include this construct while we target OpenMP 4.0 for more complete error handling capabilities. In future, we are investigating an error codes proposal that follows the characteristic error handling in $\mathrm{C}$ and Fortran and a callback proposal that provides at least a partial cooperative model.

We still have issues to resolve for the 4.0 proposals. We are exploring a minimal set of required cancellation points. We also must integrate the proposals into the overall standard. For example, barriers can cause deadlocks in the presence of exceptions when 
all threads besides the one that catches an exception wait at a barrier. This behavior is technically non-conforming code; we must resolve this inconsistency, possibly through the minimal cancellation point set. Overall, we will continue to explore these issues and to design solutions that will provide a complete OpenMP error-handling model.

\section{References}

1. Compaq Computer Corporation. Compaq Fortran - Language Reference Manual, September 1999. Order number AA-Q66SD-TK.

2. J.S. Danaher, I.-T. Angelina Lee, and C.E. Leiserson. Programming with Exceptions in JCilk. Science of Computer Programming, 63(2):147-171, December 2006.

3. A. Duran, R. Ferrer, J.J. Costa, M. Gonzàlez, X. Martorell, E. Ayguadé, and J. Labarta. A Proposal for Error Handling in OpenMP. Intl. Journal of Parallel Programming, 35(4):393416, August 2007.

4. P. Becker (editor). Working Draft: Standard for Programming Language $\mathrm{C}++$, November 2009. Document number N3000.

5. IEEE. Threads Extension for Portable Operating Systems (Draft 6), February 1992. Document P1003.4a/D6.

6. Intel Corporation. Intel Threading Building Blocks Reference Manual. Technical report, July 2009. Document number 315415-003US.

7. V. Issarny. An Exception Handling Model for Parallel Programming and its Verification. In Proc. of the Conf. on Software for Citical Systems, pages 92-100, New Orleans, LA, USA, December 1991.

8. M. Klemm, R. Veldema, M. Bezold, and M. Philippsen. A Proposal for OpenMP for Java. In Proc. of the Intl. Workshop on OpenMP, Reims, France, June 2006. No page numbers.

9. J.R. Larus and R. Rajwar. Transactional Memory (Synthesis Lectures on Computer Architecture). Morgan \& Claypool Publishers, January 2007.

10. M. Milovanović, R. Ferrer, O. Unsal, A. Cristal, X. Martorell, E. Ayguadé, J. Labarta, and M. Valero. Transactional Memory and OpenMP. In Proc. of the 3rd Intl. Workshop on OpenMP: Practical Programming Model for the Multi-Core Era, pages 37-53, Beijing, China, June 2007. LNCS 4935.

11. MPI Forum. MPI: Extensions to the Message-passing Interface, Version 2.2. Technical report, MPI Forum, September 2009.

12. S. Oaks and H. Wong. Java Threads. O'Reilly, Sebastopol, CA, USA, 3rd edition, 2004.

13. OpenMP ARB. OpenMP Application Program Interface, v. 3.0, May 2008.

14. M. Süß and C. Leopold. Implementing Irregular Parallel Algorithms with OpenMP. In Proc. of the Euro-Par 2006 Conf., pages 635-644, Dresden, Germany, August 2006.

15. I. Sommerville. Software Engineering. Pearson Education, Ltd., Harlow, UK, 8th edition, April 2007.

16. N. Stoughton. POSIX Liaison Report, February 2008. Document number N2536.

17. S. Tazuneki and T. Yoshida. Concurrent Exception Handling in a Distributed Object-Oriented Computing Environment. In Proc. of the 7th Intl. Conf. on Parallel and Distributed Systems Workshops, pages 75-82, Iwate, Japan, July 2000.

18. The Open Group. The Open Group Base Specifications Issue 7, December 2008. IEEE Std 1003.1-2008 and POSIX.1-2008.

19. J. Xu, A. Romanovsky, and B. Randell. Concurrent Exception Handling and Resolution in Distributed Object Systems. IEEE Transactions on Parallel and Distributed Systems, 11(10):1019-1032, October 2000. 